\title{
A square root pattern of changes in heart rate variability during the first year after circumferential pulmonary vein isolation for paroxysmal atrial fibrillation and their relation with long-term arrhythmia recurrence
}

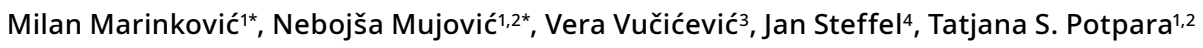 \\ 1 Department of Cardiology, Clinical Center of Serbia, Belgrade, Serbia \\ 2 School of Medicine, University of Belgrade, Belgrade, Serbia \\ 3 Center for Anesthesiology and Reanimatology, Clinical Center of Serbia, Belgrade, Serbia \\ 4 Department of Cardiology, University Hospital Zurich, Zurich, Switzerland
}

\section{KEY WORDS \\ ablation for atrial fibrillation, atrial fibrillation recurrence, heart rate variability, Holter monitoring, pulmonary vein isolation}

\section{EDITORIAL}

by Shantsila, Gupta, Lip, see p. 179

Correspondence to:
Tatjana S. Potpara, MD, PhD,
Department of Cardiology,
Clinical Center of Serbia,
Višegradska 26, 11000 Belgrade,
Serbia, phone: +381600560992,
email: tanjapotpara@gmail.com
Received: December 16, 2019.
Revision accepted:
February 12, 2020.
Published online:
February 12, 2020.
Kardiol Pol. 2020; 78 (3): 209-218
doi:10.33963/KP.15187
Copyright by the Author(s), 2020
* MM and NM contributed
equally to this work.

\begin{abstract}
BACKGROUND An incidental lesion of the parasympathetic ganglia during circumferential pulmonary vein isolation (CPVI) may affect heart rate variability (HRV).

AIMS We studied the pattern of changes in HRV parameters and the relationship between the 1-year HRV change following CPVI and the recurrence of atrial fibrillation (AF).

METHODS A total of 100 consecutive patients undergoing CPVI for paroxysmal AF were enrolled (mean [SD] age, 56 [11.2] years; 61 men). We measured HRV on the day before and after CPVI, and then at 1 month as well as 3, 6, and 12 months after CPVI using 24-hour Holter monitoring.

RESULTS During the median follow-up of 33 months, 38 patients experienced the late recurrence of AF (LRAF). Compared with the pre-CPVI values, HRV was significantly attenuated on day 1 after CPVI in all patients. However, at 3 to 6 months after CPVI, all HRV parameters remained significantly decreased in LRAF-free patients but not in those with LRAF. The multivariate Cox analysis showed that early AF recurrence within the blanking period (hazard ratio $[\mathrm{HR}], 4.87 ; 95 \% \mathrm{CI}, 2.44-9.69 ; P<0.001$ ) and a change in the standard deviation of normal-to-normal intervals (SDNN) observed 3 months after ablation (HR, 0.99; 95\% CI, 0.98-1; $P=0.01$ ) were associated with LRAF. The cumulative LRAF freedom after CPVI was greater in patients with an SDNN reduction of more than $25 \mathrm{~ms}$ reported 3 months after ablation than in those with a reduction of $25 \mathrm{~ms}$ or lower (log-rank $P=0.004$ ).

CONCLUSIONS Sustained parasympathetic denervation during 12 months after CPVI was a marker of successful CPVI, whereas a 3-month post-CPVI SDNN reduction of $25 \mathrm{~ms}$ or lower predicted LRAF.
\end{abstract}

INTRODUCTION Autonomic nervous system significantly modulates the triggers for atrial fibrillation (AF) within the pulmonary veins (PVs). ${ }^{1}$ Circumferential pulmonary vein isolation (CPVI) is the mainstay of catheter ablation (CA) for paroxysmal AF (PAF); however, arrhythmia reoccurs in up to $30 \%$ to $50 \%$ of patients with $\mathrm{AF}$ undergoing $\mathrm{CA}$ for the first time. $^{2}$

The CPVI procedure may affect the cardiac autonomic nervous activity through (un)intentional injury of the left atrial (LA) parasympathetic ganglia (ganglionated plexi [GP]) in the proximity of the PVs by radiofrequency (RF) current. ${ }^{1,3}$ 


\section{WHAT'S NEW?}

Circumferential pulmonary vein isolation may affect the cardiac autonomic nervous activity through an incidental lesion of the left atrial parasympathetic ganglia. Currently available data on the long-term pattern of ablation-related cardiac denervation and impact on the ablation outcome are conflicting. In our study, we observed a characteristic square root pattern of the longitudinal change in heart rate parameters in the first year after ablation. The change in heart rate variability, indicating a significant parasympathetic denervation, persisted throughout the first 12 months after the procedure in arrhythmia-free patients. The parameters returned to baseline values in patients with a relapse. A multivariable analysis showed that a reduced standard deviation of normal-to-normal intervals at the end of the 3-month postablation blanking period (by more than $25 \mathrm{~ms}$ compared with the preablation value) was significantly associated with long-term freedom from atrial fibrillation.

The LA denervation is reported to significantly reduce the $\mathrm{AF}$ recurrence rate after $\mathrm{CPVI} .{ }^{2,4,5}$ However, in the months following CA, the processes of healing and reinnervation ensue, potentially influencing the procedure outcome. ${ }^{3,6,7}$ A long-term pattern of CA-related cardiac denervation (and subsequent reinnervation) is underreported and currently available data are conflicting. ${ }^{3,6-8}$ We studied the pattern of longitudinal changes in heart rate variability (HRV; as a measure of cardiac denervation) after CPVI for PAF as well as the relationship between the post-CPVI change in $\mathrm{HRV}$ and long-term freedom from AF.

METHODS Study population Patient selection for this prospective single-center study is presented in Supplementary material, Figure S1. Of the 294 consecutive patients who underwent 445 RF-CA procedures for symptomatic and drug-resistant AF in the Clinical Centre of Serbia between 2013 and 2016, we excluded those after ablation for persistent $\mathrm{AF}$, repeat ablation for $\mathrm{AF}$, and / or substrate-based ablation for AF $(n=195)$; those with a history of slow pathway or septal accessory pathway ablation and / or pacemaker implantation ( $n=23)$; those with a history of heart failure, myocardial infarction (MI), and / or diabetes mellitus ( $n=69$ ); those with more than $20 \%$ of the 24-hour Holter monitoring time spent in $\mathrm{AF}$, frequent atrial and / or ventricular premature beats or artifacts $(n=49)$; and those with 12 months or less of post-CA follow-up $(n=9)$.

The study group included 100 patients with PAF with no significant structural heart disease who underwent their first CA for AF with CPVI stand-alone ablation strategy and were followed for more than 12 months after the procedure. We defined PAF as an AF episode lasting longer than 30 seconds but shorter than 7 days. ${ }^{2}$ All antiarrhythmic drugs (AADs) were discontinued at least 5 half-lives before CA (amiodarone was ceased more than 3 months prior to $C A$ ). Before the procedure, all patients underwent transthoracic echocardiography, cardiopulmonary exercise testing, and 24-hour Holter monitoring. The study was approved by the institutional ethics committee, and all patients signed informed consent to participate in the study.

Ablation procedure The CPVI procedure in our hospital was described in detail elsewhere. ${ }^{9}$ Briefly, after transseptal puncture, a 3-dimensional map was created by the electroanatomic mapping system (EnSite Velocity, St. Jude, Minneapolis, Minnesota, United States) and thereafter fused with the segmented computed tomography model of the LA. The RF current was applied via an externally irrigated 4-mm tip catheter (Therapy Cool-Flex, St. Jude), with a power of $30 \mathrm{~W}$ and a flow rate of $17 \mathrm{ml} / \mathrm{min}$. Local $\mathrm{RF}$ delivery was continued only until a reduction of atrial potential amplitude greater than $80 \%$ was achieved or for a maximum of 30 to 40 seconds and 40 to 60 seconds at the posterior and anterior LA wall, respectively, whichever came first. Ipsilateral PVs (left and right) were encircled in pairs with a circumferential ablation line deployed $5 \mathrm{~mm}$ from the anterior and 15 to $20 \mathrm{~mm}$ from the posterior aspects of their ostia, respectively. During ablation, the electrical activity of PVs was evaluated with a circumferential mapping catheter. ${ }^{9}$ In patients with a history of typical atrial flutter, ablation of the cavotricuspid isthmus was performed during the same session. ${ }^{2}$

Postablation follow-up We routinely administered AADs during the 3-month blanking peri$\mathrm{od}^{2}$ and thereafter discontinued them in all patients. Postablation follow-up visits were scheduled at 1 month, 3 months, 6 months, and every 6 months afterwards. They included physical examination, 12-lead electrocardiography, and 24-hour Holter monitoring. The recurrence of AF was defined as any symptomatic or asymptomatic AF or atrial tachycardia (AT) episode longer than 30 seconds. ${ }^{2}$ The early recurrence of AF (ERAF) was defined as atrial arrhythmia recurring within the 3 -month blanking period after CA, whereas the recurrence of arrhythmia 3 months after $C A$ was considered the late recurrence of AF (LRAF). ${ }^{2}$ Patients experiencing ERAF during the blanking period were treated by cardioversion or rate control (but without early reablation), and those with LRAF after the blanking period were offered AAD readministration or repeat $C A{ }^{2}$

Analysis of heart rate variability In all patients, serial 3-channel 24-hour Holter recordings (Del Mar Reynolds Medical, Inc., Irvine, California, United States) were obtained for HRV analysis with the Impresario software (Del Mar Reynolds Medical, Inc.) 1 day prior to ablation as well as 1 day, 1 month, 3 months, and 6 months after the procedure, and every 
6 months thereafter. To provide high-quality HRV measurements, all patients with any AF episodes on Holter recordings, ventricular and / or atrial premature beats, and / or artifacts lasting more than $20 \%$ of the recording time were excluded from further analysis. ${ }^{10}$ The following HRV time parameters were analyzed: the standard deviation of normal-to-normal (NN) intervals (SDNN; ms), the root mean square of the differences between adjacent NN intervals (rMSSD; ms), the standard deviation of differences between adjacent NN intervals (SDSD; ms), the HRV index (the integral of the density of the R-R interval histogram divided by its height), mean R-R interval (ms), mean heart rate (HR; bpm), and the percentage of adjacent $\mathrm{NN}$ interval differences greater than $50 \mathrm{~ms}$ (pNN50; \%). ${ }^{10}$ The HRV frequency measures were calculated with the fast Fourier transform for each 5-minute segment of data with the low- to high-frequency (LF/HF) ratio. ${ }^{10}$

Statistical analysis Continuous variables with normal distribution were presented as mean (SD), and those with asymmetrical distribution, as median and interquartile range (IQR; first quartile, third quartile [Q1-Q3]). Categorical variables were presented as percentages. Continuous variables were compared by the independent sample $t$ test or the Mann-Whitney test, as appropriate, while the $\mathrm{X}^{2}$ test and Fisher test were used to compare proportions. Continuous variables (the HRV parameters) with repeated measures (successive Holter recordings) were analyzed with the Friedman test, and then the post hoc analysis was conducted using the Wilcoxon test with the Bonferroni correction. Risk factors for LRAF were evaluated using the univariate and multivariate Cox regression analyses. The cutoff value of continuous variables significantly associated with LRAF was determined by the receiver operating characteristic curve analysis. The LRAF-free survival rates were compared using the Kaplan-Meier analysis with the log-rank test. A 2-sided $P$ value of less than 0.05 was considered significant. All analyses were performed with the SPSS software, version 21.0 (IBM Corp., Armonk, New York, United States).

RESULTS Study population The study group included 100 patients (mean [SD] age, 56 [11.2] years; 61 men). Baseline clinical characteristics are presented in TABLE 1.

The mean (SD) left and right CPVI ablation line was 116 (19) $\mathrm{mm}$ and 119 (22) $\mathrm{mm}$ long, respectively. The CPVI was successfully completed in all patients with the mean (SD) RF delivery time for left and right $\mathrm{PV}$ isolation of 29.1 (14.6) min and 31.2 (14.4) min, respectively. Ablation of typical atrial flutter was performed in
42 patients. Three major complications (inguinal hematoma, retroperitoneal hematoma, and coronary artery air embolism) occurred in 3 patients but did not impede the completion of CPVI.

The ERAF was reported in 15 patients (PAF in 7 patients, paroxysmal AT in 6 , and persistent AT in 2). During the 3-month blanking period, the following drugs were prescribed: propafenone (to 68 patients), amiodarone (22), sotalol (7), a $\beta$-blocker (1), and verapamil (1); 1 patient was taken off AADs due to bradycardia.

During the median post-CA follow-up of 33 months (IQR, 21-40 months), a total of 38 patients developed LRAF (PAF was present in 27 patients, paroxysmal AT in 9, and persistent AT in 2). In 17 patients, LRAF was noted between 3 and 12 months after $C A$, whereas in the remaining 21 patients, the recurrence of arrhythmia was observed later than 12 months after CA. During the first year after $\mathrm{CA}$, in 16 of the 17 patients with LRAF, AADs were reinstituted as monotherapy with propafenone $(n=1)$, sotalol $(n=1)$, verapamil $(n=1)$, or a $\beta$-blocker $(n=5)$, or as a combination of amiodarone and a $\beta$-blocker $(n=3)$ or propafenone and a $\beta$-blocker $(n=5)$. The remaining asymptomatic patient continued without AADs. The CA procedure was repeated in 18 of the 38 patients with LRAF after a mean (SD) time of 16 (7) months. At repeat CA, PV reconnections were identified in 16 of the $18 \mathrm{pa}$ tients (89\%) with a mean (SD) number of $2.4(0.9)$ reconnected PVs per patient. The PV reconnection rates (per patient) were as follows: left superior PV, 10/18 (56\%); left inferior PV, 7/18 (39\%); right superior PV, 11/18 (61\%); and right inferior PV, 10/18 (56\%).

No new adverse cardiovascular events such as sudden death, acute coronary syndrome, or cardiac decompensation were observed during the follow-up.

\section{Changes in heart rate variability parameters} at 1 year after ablation There were no significant differences in $\mathrm{HRV}$ parameters at baseline (before CA) in patients with LRAF after CA and those without, except for SDNN (median, $117 \mathrm{~ms}$ [IQR, 99-145 ms] vs $141 \mathrm{~ms}$ [IQR, 108-201 ms]; $P=0.04$; TABLE2).

The longitudinal changes in HRV at 1 year after ablation are summarized in TABLE 2 and FIGURES 1 and 2. There was a significant decrease in SDNN, rMSSD, SDSD, HRV index, mean R-R interval, pNN50, and LF/HF ratio, as well as a significant increase in the mean $\mathrm{HR}$ after $\mathrm{CA}$ compared with the preablation values, both in patients with LRAF (all $P<0.001$ ) and those without (all $P<0.001$; except for changes in SDSD and the LF / HF ratio with a $P$ value of 0.02 and 0.002 , respectively). In all patients (ie, those with and without LRAF), the post hoc analysis showed a significant attenuation of all HRV parameters on the first day 
TABLE 1 Baseline clinical characteristics of the study population

\begin{tabular}{|c|c|c|c|c|}
\hline Variable & $\begin{array}{l}\text { All patients } \\
(n=100)\end{array}$ & $\begin{array}{l}\text { Patients with } \\
\text { LRAF }(n=38)\end{array}$ & $\begin{array}{l}\text { Patients without } \\
\text { LRAF }(n=62)\end{array}$ & $P$ value \\
\hline Age, y, mean (SD) & $56.0(11.2)$ & $57.5(9.5)$ & $55.1(12.1)$ & 0.29 \\
\hline Male sex & $61(61)$ & $23(60.5)$ & $38(61.3)$ & 0.94 \\
\hline BMI, $\mathrm{kg} / \mathrm{m}^{2}$, mean (SD) & $27.1(4.2)$ & $27.1(4.6)$ & $27.1(3.5)$ & 0.99 \\
\hline Years since first-diagnosed AF, median (IQR) & $4(2-9)$ & $3(2-7.3)$ & $5(2.5-9.5)$ & 0.28 \\
\hline Duration of an AF episode (>48 h) & $21(21)$ & $11(28.9)$ & $10(16.1)$ & 0.13 \\
\hline LVEDD, mm, mean (SD) & $51.9(4)$ & $52.0(4.5)$ & $51.8(3.7)$ & 0.87 \\
\hline LVEF, \%, mean (SD) & $62.2(6.5)$ & $60.7(7.7)$ & $63.1(5.5)$ & 0.08 \\
\hline LA diameter, mm, mean (SD) & $39.4(5.2)$ & $39.0(5.6)$ & $39.7(4.9)$ & 0.54 \\
\hline CAD (with no history of MI) & $3(3)$ & $3(7.9)$ & 0 & 0.05 \\
\hline Hypertension & $49(49)$ & $19(50)$ & $30(48.4)$ & 0.88 \\
\hline Chronic pulmonary disease & $2(2)$ & $2(5.3)$ & 0 & 0.14 \\
\hline History of stroke & $9(9)$ & $1(2.6)$ & $8(12.9)$ & 0.15 \\
\hline History of hyperthyroidism & $9(9)$ & $2(5.3)$ & $7(11.3)$ & 0.48 \\
\hline $\mathrm{CHA}_{2} \mathrm{DS}_{2}$-VASc score, mean (SD) & $1.5(1.4)$ & $1.3(1.1)$ & $1.6(1.5)$ & 0.37 \\
\hline HATCH score, mean (SD) & $0.7(0.8)$ & $0.6(0.8)$ & $0.8(0.9)$ & 0.41 \\
\hline Patients with complete PVI & $100(100)$ & $38(100)$ & $62(100)$ & $>0.99$ \\
\hline Cavotricuspid isthmus ablation & $42(42)$ & $11(29.7)$ & $31(50)$ & 0.048 \\
\hline Fluoroscopy time, min, mean (SD) & $29.7(14.4)$ & $33.2(16)$ & $27.7(13.1)$ & 0.07 \\
\hline RF delivery time, min, mean (SD) & $64.2(29.2)$ & $67.1(32.5)$ & $62.5(27.3)$ & 0.46 \\
\hline Ineffective AADs, mean (SD) & $2.6(1.3)$ & $2.5(1.3)$ & $2.6(1.3)$ & 0.72 \\
\hline Amiodarone & $58(58)$ & $24(63.2)$ & $34(54.8)$ & 0.41 \\
\hline$\beta$-Blocker & $65(65)$ & $24(63.2)$ & $41(66.1)$ & 0.76 \\
\hline ACEIs / ARBs & $39(39)$ & $28(73.7)$ & $11(17.7)$ & $<0.001$ \\
\hline Statins & $22(22)$ & $10(26.3)$ & $12(19.7)$ & 0.44 \\
\hline eGFR, ml/min $/ 1.73 \mathrm{~m}^{2}$, mean (SD) & $104.1(33)$ & $107.3(31.4)$ & $106.7(34.7)$ & 0.52 \\
\hline CRP after ablation, mg/l, median (IQR) & $9.7(6.5-16.9)$ & $10.1(6.6-18.5)$ & $9.6(5.9-14.1)$ & 0.47 \\
\hline ERAF & $15(15)$ & $13(34.2)$ & $2(3.2)$ & $<0.001$ \\
\hline
\end{tabular}

Data are presented as number (percentage) unless otherwise indicated.

Abbreviations: $A A D s$, antiarrhythmic drugs; $A C E I$, angiotensin-converting enzyme inhibitor; $A F$, atrial fibrillation; $A R B$, angiotensin receptor blocker; $\mathrm{BMI}$, body mass index; $\mathrm{CAD}$, coronary artery disease; $\mathrm{CHA}_{2} \mathrm{DS}_{2}$-VASc, congestive heart failure, hypertension, age $\geq 75$ years, diabetes mellitus, history of stroke or thromboembolism, vascular disease, age 65 to 74 years, female sex; CRP, C-reactive protein; eGFR, estimated glomerular filtration rate; ERAF, early recurrence of atrial fibrillation; HATCH, hypertension, age, stroke or transient ischemic attack, chronic obstructive pulmonary disease, heart failure; IQR, interquartile range; LA, left atrial; LRAF, late recurrence of atrial fibrillation; LVEDD, left ventricular end-diastolic dimension; LVEF, left ventricular ejection fraction; MI, myocardial infarction; PVI, pulmonary vein isolation; RF, radiofrequency

after ablation compared with the preablation values, with subsequent gradual return to the baseline values. In patients with LRAF, the postablation values of rMSSD, SDSD, HRV index, pNN50, LF / HF ratio, and SDNN returned to the values similar to baseline (rMSSD and SDSD at 1 month; HRV index, pNN50, and $\mathrm{LF} / \mathrm{HF}$ ratio at the Holter recording at 3 months after CA; and SDNN at 6 months after $(A)$, whereas the mean R-R interval and mean HR remained significantly reduced and increased, respectively (compared with the preablation values) at 1 year after CA. However, in LRAF-free patients, all HRV parameters (except the LF / HF ratio) remained significantly changed on serial Holter recordings during the first year after the procedure, as presented in TABLE 2 and FIGURES 1 and 2.

The comparison of changes in the HRV parameters during the first year after CA between patients with and without LRAF is shown in TABLE3. The postablation reduction of several HRV 


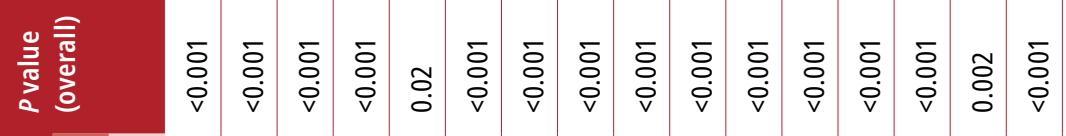

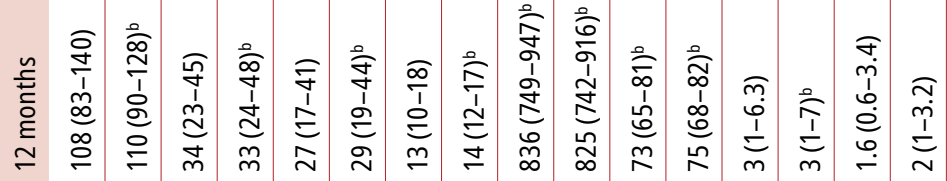

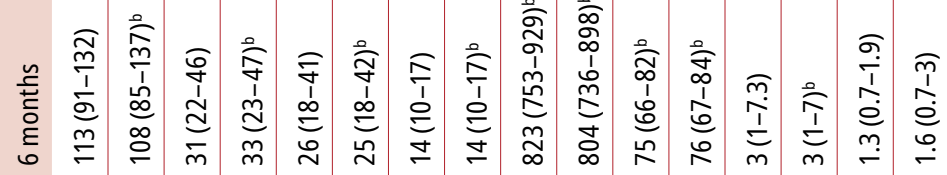

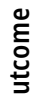

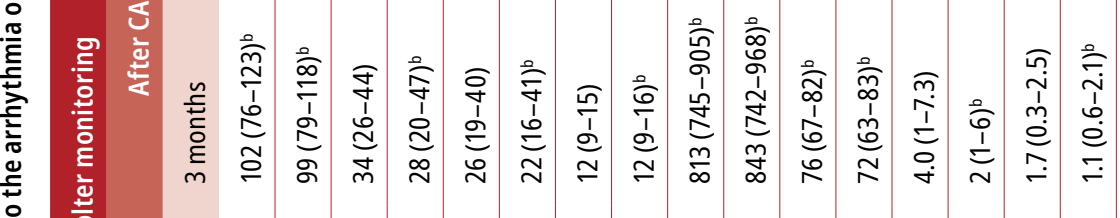

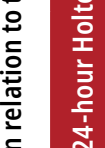

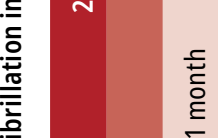

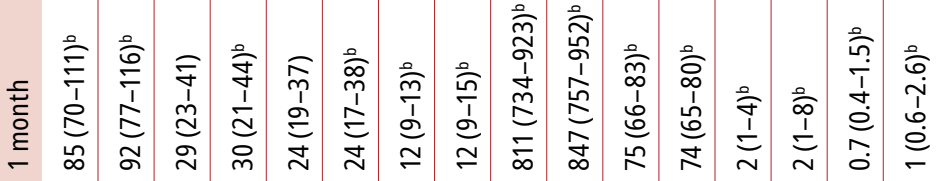

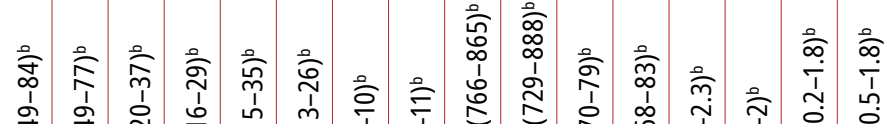

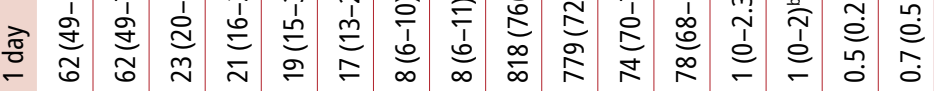

$\stackrel{\varpi}{\stackrel{\varpi}{\varpi}}$

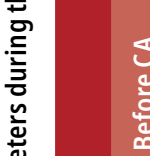

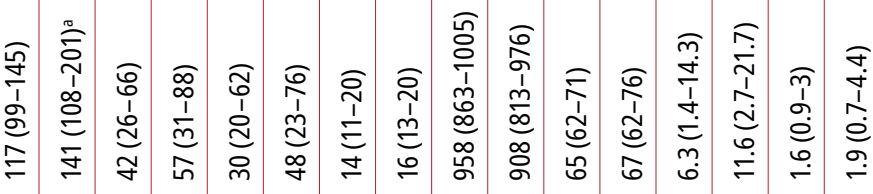

高

高

을

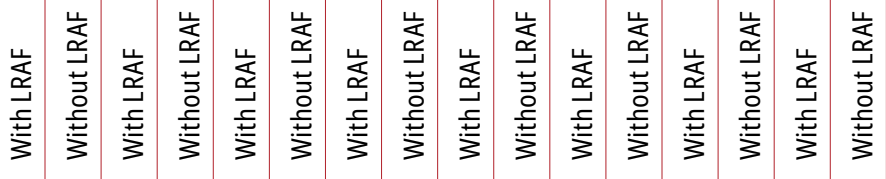

蛋

๘

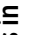

莺

U

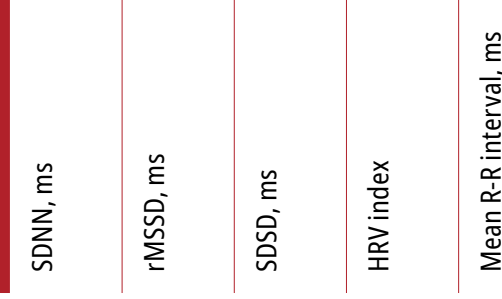

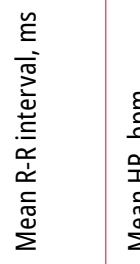

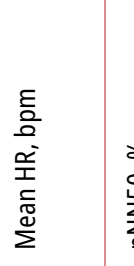



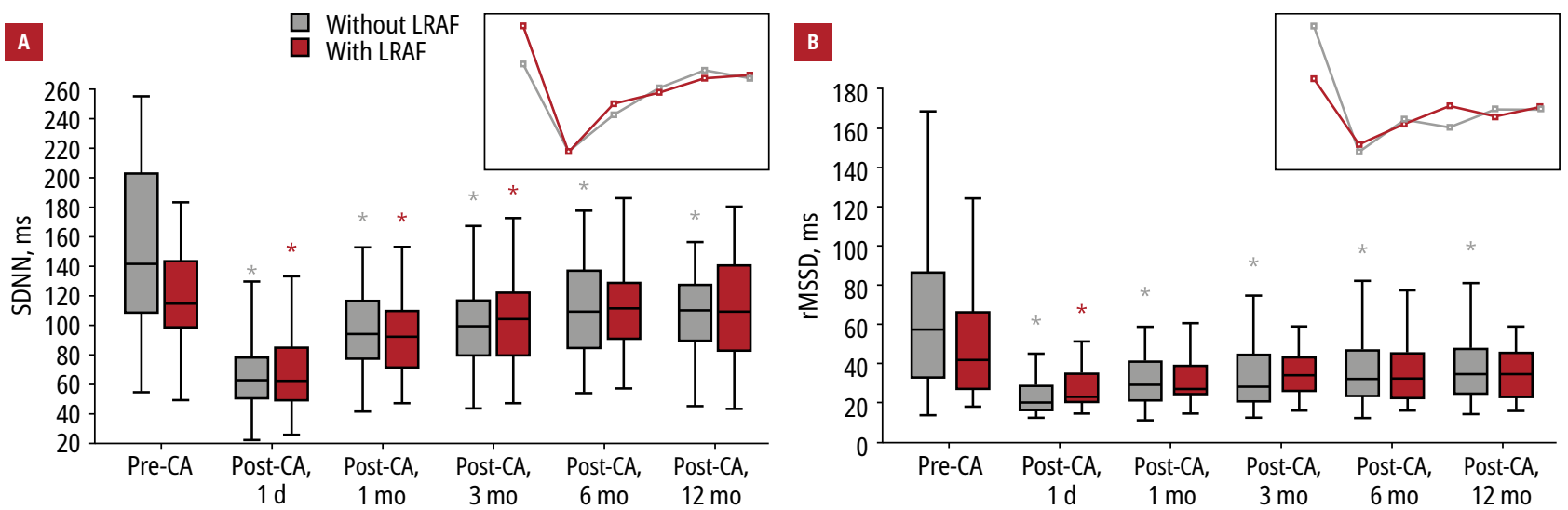

c

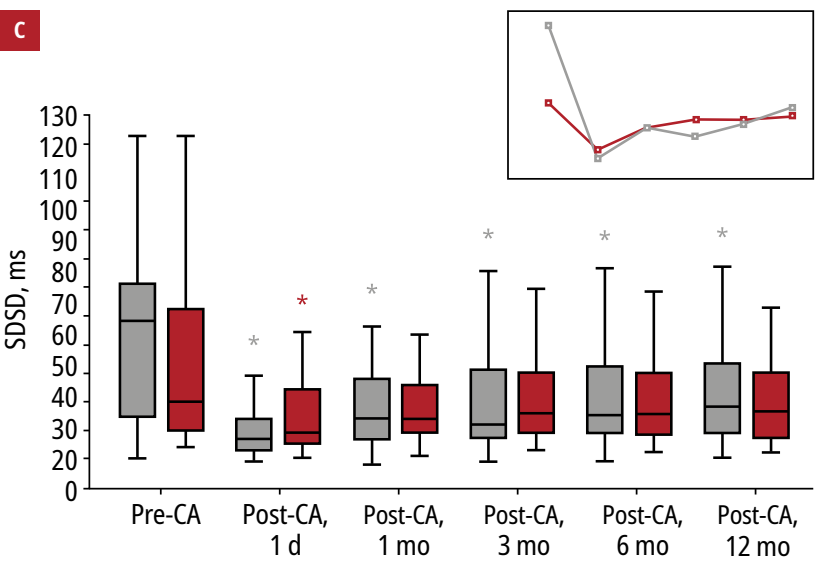

D

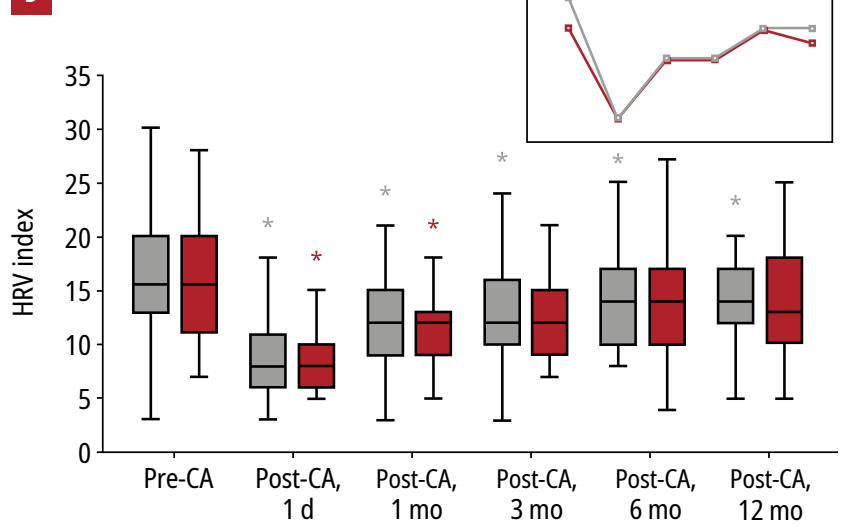

FIGURE 1 Box-and-whisker plots illustrating the square root pattern of changes in heart rate variability (HRV) observed on 24-hour Holter recordings during the first year after catheter ablation (CA): A - standard deviation of normal-to-normal intervals (SDNN); B - root mean square of the differences between adjacent normal-to-normal intervals (rMSSD); $\mathbf{C}$ - standard deviation of differences between adjacent normal-to-normal intervals (SDSD); $\mathbf{D}-\mathrm{HRV}$ index. The $\boldsymbol{P}$ value was considered significant according to the Bonferroni correction for the post hoc analysis. Grey asterisks represent the significant $P$ value for the post hoc analysis in patients without the late recurrence of atrial fbrillation (LRAF). Red asterisks represent the significant $P$ value for the post hoc analysis in patients with LRAF.

parameters (ie, $\triangle \mathrm{SDNN}, \triangle \mathrm{rMSSD}, \triangle \mathrm{SDSD}$, and $\triangle$ pNN50) was significantly greater in the LRAF-free patients compared with those with LRAF on the first postablation day, and a significant difference in these HRV parameters between both groups was mostly preserved at 1 month as well as at 3, 6, and 12 months after the procedure (TABLE3). There were no significant differences in the postablation changes in other HRV parameters (ie, $\Delta \mathrm{HRV}$ index, $\Delta$ mean $\mathrm{R}-\mathrm{R}$ interval, $\Delta$ mean $\mathrm{HR}$, and $\Delta \mathrm{LF} / \mathrm{HF}$ ratio) during the first year after $C A$ between patients with LRAF and those without.

In patients with LRAF $(\mathrm{n}=38)$, there was no difference in the change of the HRV parameters between patients who underwent the redo-CA procedure and those who were treated only with AADs (median SDNN before CA, $113 \mathrm{~ms}$ [IQR, 95-150 ms] vs $122 \mathrm{~ms}$ [IQR, 102-143 ms], $P=0.5$; reduction in the median SDNN at 3 months after CA, $5 \mathrm{~ms}$ [IQR, -5 to $38 \mathrm{~ms}$ ] vs $21 \mathrm{~ms}$ [2-46 ms], $P=0.23$ ).

Risk factors for the late recurrence of atrial fibrillation The univariate Cox analysis included all variables listed in TABLES 1-3. It showed a significant univariate association between
LRAF and the following variables: ablation for the cavotricuspid isthmus, ERAF, pre-CA SDNN, $\triangle$ SDNN at 1 day, 1 month, and 3 months postCA, $\triangle$ rMSDD at 3 months post-CA, and $\triangle$ SDSD at 1 day post-CA (all $P<0.05$ ). However, on multivariate analysis, only ERAF (hazard ratio [HR], 4.87; 95\% CI, 2.44-9.69; $P<0.001)$ and $\triangle$ SDNN at 3 months post-CA (HR, 0.99; 95\% CI, 0.98$1 ; P=0.01$ ) were independently associated with the late arrhythmia relapse (see Supplementary material, Table S1).

The reduction of $\triangle$ SDNN at 3 months post-CAshowed a predictive value for LRAF (area under the receiver operating characteristic curve, $0.63 ; 95 \% C I, 0.52-0.74 ; P=0.03$ ). The cutoff value of $25 \mathrm{~ms}$ or lower for $\triangle$ SDNN at 3 months post-CA had the best predictive ability for LRAF (HR, 2.52; 95\% CI, 1.30-4.89; $P=0.01)$ with a sensitivity and specificity of $62.9 \%$ and $63.2 \%$, respectively. The $\mathrm{Ka}$ plan-Meyer cumulative probability of post-CA LRAF-freedom was significantly higher in patients with a reduction in $\triangle$ SDNN at 3 months post-CA higher than $25 \mathrm{~ms}$ than in those with a reduction of $25 \mathrm{~ms}$ or lower (log-rank $P=0.004$ ) (FIgURE 3$)$. 

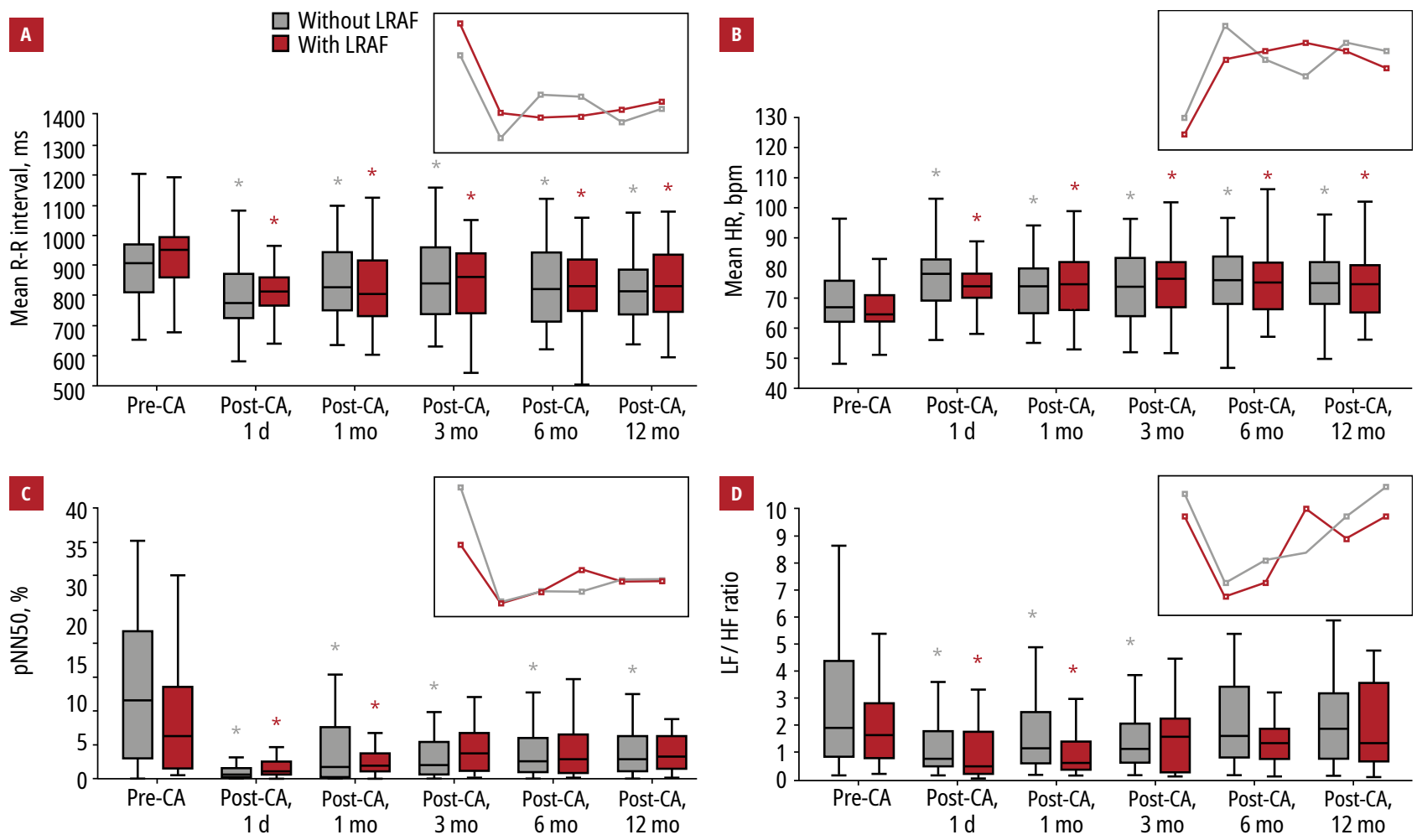

FIGURE 2 Box-and-whisker plots depicting the square root pattern of changes in heart rate variability (HRV) observed on 24-hour Holter recordings during the first year after catheter ablation (CA): A - mean R-R interval; B - mean heart rate (HR); C - percentage of adjacent normal-to-normal interval differences greater than $50 \mathrm{~ms}$ (pNN50); D - low- to high-frequency (LF/HF) ratio. The $P$ value was considered significant according to the Bonferroni correction for the post hoc analysis. Grey asterisks represent the significant $P$ value for the post hoc analysis in patients without the late recurrence of atrial fbrillation (LRAF). Red asterisks represent the significant $P$ value for the post hoc analysis in patients with LRAF.

Abbreviations: bpm, beats per minute

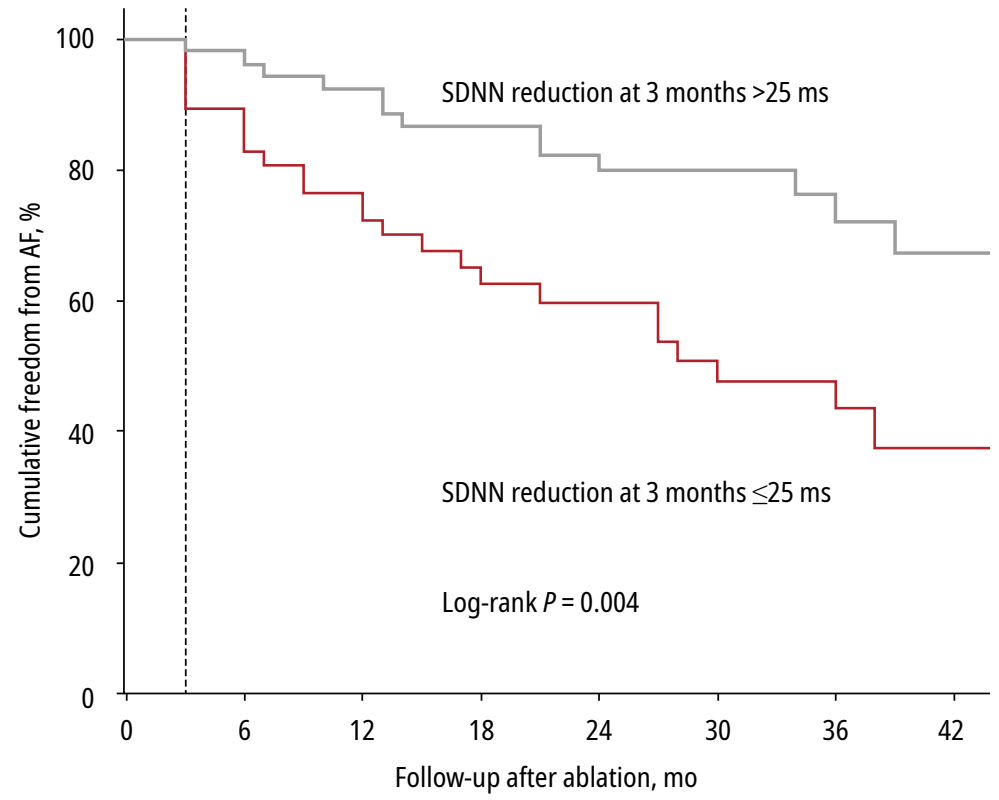

FIGURE 3 Kaplan-Meier curves of freedom from atrial fibrillation (AF) after ablation in relation to the change in the standard deviation of normal-to-normal intervals (SDNN) at 3 months after ablation

$71.4 \%$ to $100 \%$ in those who experienced ERAF $(\mathrm{n}=15)$ (Supplementary material, Figure S2).

DISCUSSION Our study of 100 patients with PAF with no significant structural heart disease showed a square root pattern of longitudinal change in the HRV parameters during the first year after CPVI, with an immediate postablation change in HRV indicating a significant parasympathetic attenuation and subsequent (partial) gradual return of the HRV indices in the following months toward preablation levels. The HRV markers of a significant reduction in parasympathetic tone persisted throughout the next 12 months after the procedure in the arrhythmia-free patients. In patients with LRAF, the HRV parameters returned to baseline values within 3 to 6 months after CPVI. The change in SDNN at the end of the 3-month post-CPVI blanking period was significantly associated with long-term $\mathrm{AF}$ recurrence.

A reduction of $25 \mathrm{~ms}$ or lower for $\triangle$ SDNN at 3 months post-CA was associated with an increase in the prevalence of LRAF from $19.6 \%$ to $41 \%$ in the ERAF-free patients $(n=85)$ and from

Autonomic nervous system and atrial fibrillation The LA contains 4 GPs, which are located at the epicardial aspect of the LA-PV junction. The GPs include both adrenergic and cholinergic nerve fibers that control cardiac 


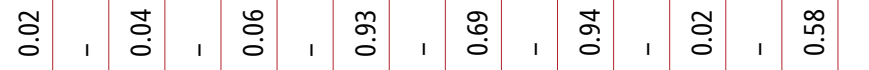

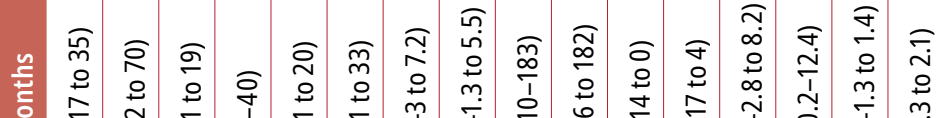

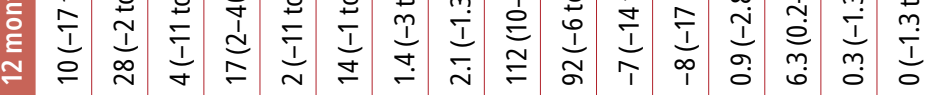

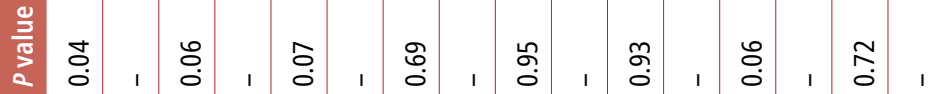

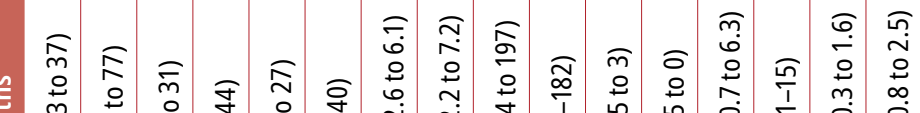

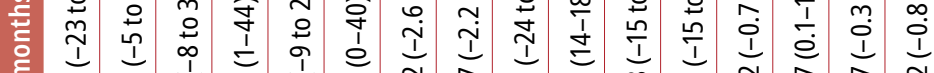

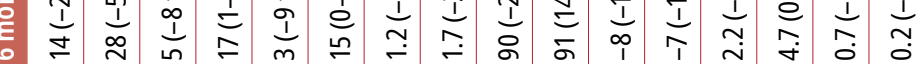

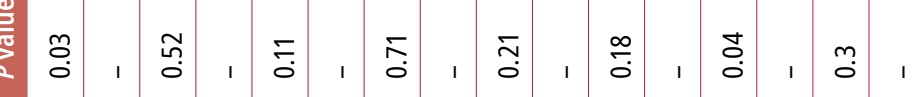

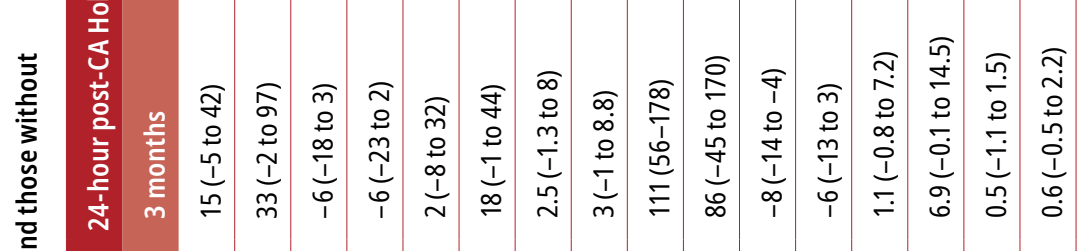

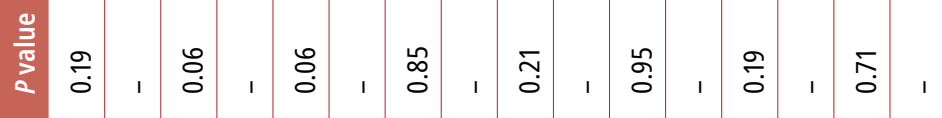

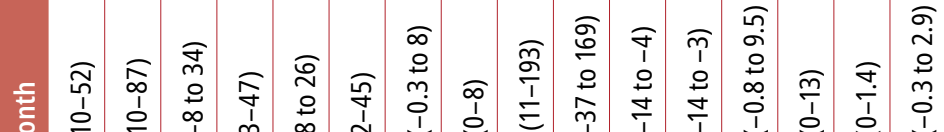

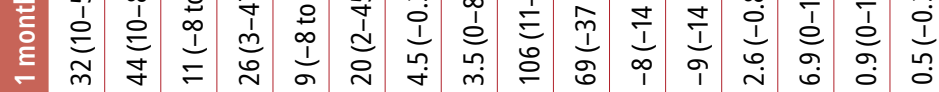

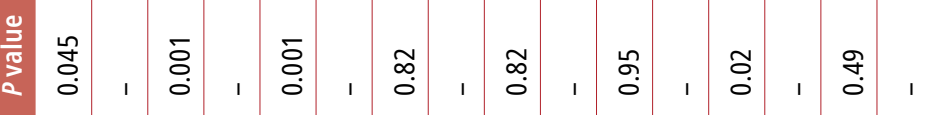

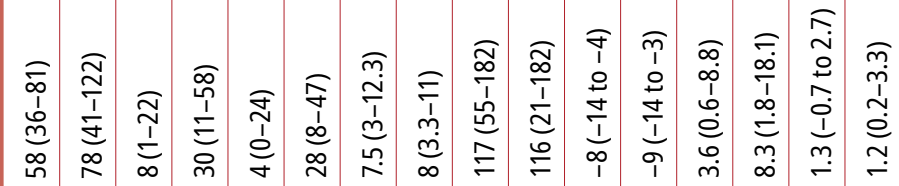


impulse formation and propagation, and therefore modulate the interplay between the PV triggers and LA substrate. ${ }^{6}$ Thus, in younger patients with no evidence of structural heart disease, vagal predominance promotes the triggered PV activity associated with afterdepolarization, shortens the PV refractoriness and AF cycle length, resulting in the inducibility and / or maintenance of AF., ${ }^{1,11}$

Outcomes of denervation and ablation for atrial fibrillation The GP ablation led to a vagal response (ie, bradycardia and hypotension) during RF current application, and subsequently to noninducibility of vagally mediated AF. ${ }^{1}$ Several studies reported a significant change of the HRV parameters after CPVI, indicating a remarkable parasympathetic withdrawal. ${ }^{3,6-8,12,13}$ The GPs in the LA are located close to the selected CPVI ablation trajectory ${ }^{1}$ and, therefore, are commonly collaterally damaged during the ablation. ${ }^{3,6-8,12,13}$ Clinical studies showed a significantly higher number of cases free of AF after CPVI in patients who exhibited a more pronounced postablation change in the HRV indices of parasympathetic denervation. ${ }^{3,6,7,12,13}$ Consequently, the GPs in the LA became an important adjuvant ablation target on top of CPVI, significantly improving the long-term CPVI outcome. ${ }^{4,5}$ Moreover, parasympathetic attenuation after the procedure had a supplemental and distinct effect on freedom from AF, irrespective of the late PV reconnection. ${ }^{7}$ However, healing of an RF-induced injury in the following weeks may be accompanied with reinnervation at the LA-PV junction, potentially influencing the long-term outcome after CPVI. 3,7,12,13

Interestingly, the follow-up studies on persistency of denervation after AF ablation reported conflicting findings. $3,6-8,12,13$ Initially, it was suggested that post-CPVI denervation performed with a conventional RF catheter is only a transient state followed by complete reinnervation at 6 months after ablation. ${ }^{3,8}$ However, a more recent study reported more persistent HRV changes during the first year after CPVI performed with more powerful irrigated RF ablation. ${ }^{6}$ Again, the HRV reduction during the first year after CPVI was significantly more striking and persistent in the AF-free patients than in those with recurrent $A F .{ }^{6}$ In addition, the long-term HRV change after CPVI was considerably different between patients with PAF and those without. ${ }^{6}$

Our study included a homogenous cohort of PAF patients with no significant structural heart disease who underwent stand-alone CPVI conducted with the use of irrigated RF catheters. The longitudinal change in the HRV parameters during the first year after CPVI depicted a characteristic square root pattern, consisting of significant early parasympathetic denervation after $C A$ and subsequent gradual reinnervation. Of note, despite partial reinnervation after 3 to 6 months after ablation, the HRV markers of the overall sympatho-vagal balance (SDNN, SDSD, pNN50, and mean R-R interval) and more specific HRV indices of parasympathetic activity (rMSSD and LF / HF ratio) were still significantly reduced at 1 year after CPVI in the AF-free patients but not in those with the recurrence of arrhythmia. Thus, in otherwise mostly healthy patients, persistently reduced HRV after CPVI for PAF is a marker of better long-term AF control. However, since reduced HRV was previously recognized as a marker of adverse cardiovascular outcome in patients with a history of MI, heart failure, or diabetes,${ }^{14}$ it is questionable whether the post-CPVI denervation may affect the prognosis of patients undergoing ablation for AF if they develop significant cardiometabolic comorbidities in the future. Importantly, our study patients with the median postablation follow-up of almost 3 years were free of new adverse cardiovascular events, such as sudden death, MI, or heart failure.

Predicting circumferential pulmonary vein isolation outcome with the periprocedural assessment of changes in heart rate variability Previous studies identified changes in HRV occurring immediately after CPVI as a predictor of AF recurrence. ${ }^{3,6-8,12,13,15}$ Thus, postablation findings of high $\mathrm{HF}$ and a low LF / HF ratio or that changed by more than 0.26 were all independently associated with LRAF. ${ }^{6,7}$ However, interpreting changes in the HRV frequency parameters (ie, HF and LF) is limited because their assessment depends considerably on respiration and measurement conditions. ${ }^{10}$ On the other hand, the measurement of time-domain HRV parameters is more stable and reproducible in routine practice. ${ }^{10}$ The absolute value of SDNN at 3 months after CPVI was previously identified as an independent risk factor for an AF relapse. ${ }^{8}$ However, our study further underlines the utility of the periprocedural SDNN analysis. First, we show that the PAF patients with higher preablation SDNN (as a measure of overall HRV) are the best candidates for CPVI. In addition, the SDNN reduction of $25 \mathrm{~ms}$ or lower at 3 months after CPVI was associated with a 2.5-fold increase in the probability of LRAF. Data on $\triangle$ SDNN at 3 months post-CA are usually easily available on a routine follow-up Holter recording and may distinguish between the ERAF patients at increased and low risk for subsequent LRAF. Although ERAF is one of the strongest predictors of LRAF, later AF episodes gradually subside with a resolution of CA-related LA inflammation in approximately $40 \%$ to $60 \%$ of patients with ERAF. ${ }^{2}$ The reliable prediction of the long-term CPVI 
success may optimize the selection of appropriate rhythm monitoring strategy, AADs use, and anticoagulant treatment following CA. ${ }^{16}$

Study limitations Intermittent rhythm monitoring strategy with a 24-hour Holter recording probably underestimated the AF recurrence rate after CPVI. ${ }^{2}$ In all patients, AADs were routinely prescribed during the blanking period, which possibly affected the HRV parameters after CA. ${ }^{10}$ However, our postablation strategy conformed with the current guidelines. ${ }^{2}$

The study population included highly selected, relatively young, and healthy patients with PAF. Approximately $50 \%$ of the patients were hypertensive and $5 \%$ had stable coronary artery disease, but patients with diabetes, a history of MI, and / or heart failure were excluded. Thus, the transferability of our research results to the general $\mathrm{AF}$ population undergoing $\mathrm{CA}$ is limited. In our view, notwithstanding the fact that strictly including only those with lone PAF would provide straightforward information, such patient selection minimized the influence of cardiovascular disease(s) on the HRV parameters and their changes in the first months after ablation.

The exclusion of patients whose follow-up Holter recordings showed frequent aberrant beats might have introduced a selection bias. However, such an approach ensured a good quality of HRV measurements. ${ }^{10}$ The HRV analysis was limited to 12 months after CPVI, whereas the reinnervation could have continued beyond the first year after ablation.

The association between changes in HRV and freedom from AF after CA may not necessarily be a causal relationship. Persistent changes in autonomic tone may be simply the result of a more effective (ie, transmural) RF lesion, a durable ablation line, and therefore a better CA outcome.

Conclusions Our study identified a square root pattern of changes in the HRV parameters during the first year after CPVI in patients with PAF, with substantial parasympathetic withdrawal immediately after ablation and its gradual recovery in the next months. A persistent HRV attenuation within the first 12 months after ablation for $\mathrm{AF}$ was a marker of the long-term procedural success and freedom from AF. A higher preablation SDNN value potentially identified optimal candidates for CPVI, but this will require prospective validation. Conversely, an SDNN reduction of 25 ms or less at 3 months after CPVI was a reliable predictor of a subsequent AF relapse. Preprocedural and follow-up HRV analyses could improve the prediction of LRAF in patients with AF undergoing CPVI and, therefore, complement other clinical predictors of LRAF, such as ERAF.

\section{SUPPLEMENTARY MATERIAL}

Supplementary material is available at www.mp.pl/kardiologiapolska.

\section{ARTICLE INFORMATION}

CONFLICT OF INTEREST None declared.

OPEN ACCESS This is an Open Access article distributed under the terms of the Creative Commons Attribution-NonCommercial-NoDerivatives $4.0 \mathrm{In}$ ternational License (CC BY-NC-ND 4.0), allowing third parties to download articles and share them with others, provided the original work is properly cited, not changed in any way, distributed under the same license, and used for noncommercial purposes only. For commercial use, please contact the journal office at kardiologiapolska@ptkardio.pl.

HOW TO CITE Marinković M, Mujović N, Vučićević V, et al. A square root pattern of changes in heart rate variability during the first year after circumferential pulmonary vein isolation for paroxysmal atrial fibrillation and their relation with long-term arrhythmia recurrence. Kardiol Pol. 2020; 78: 209-218. doi:10.33963/ KP.15187

\section{REFERENCES}

1 Choi EK, Zhao Y, Everett TH, et al. Ganglionated plexi as neuromodulation targets for atrial fibrillation. Journal of cardiovascular electrophysiology. 2017; 28: 1485-1491.

2 Calkins H, Hindricks G, Cappato R, et al. 2017 HRS/EHRA/ECAS/APHRS/SOLAECE expert consensus statement on catheter and surgical ablation of atrial fibrillation. Heart Rhythm. 2017; 14: e275-e444.

3 Pappone C, Santinelli V, Manguso F, et al. Pulmonary vein denervation enhances long-term benefit after circumferential ablation for paroxysmal atrial fibrillation. Circulation. 2004; 109: 327-334.

4 Katritsis DG, Pokushalov E, Romanov A, et al. Autonomic denervation added to pulmonary vein isolation for paroxysmal atrial fibrillation: a randomized clinical trial. J Am Coll Cardiol. 2013; 62: 2318-2325.

5 Pokushalov E, Romanov A, Katritsis DG, et al. Ganglionated plexus ablation vs linear ablation in patients undergoing pulmonary vein isolation for persistent/long-standing persistent atrial fibrillation: a randomized comparison. Heart Rhythm. 2013; 10: 1280-1286.

6 Kang KW, Kim TH, Park J, et al. Long-term changes in heart rate variability after radiofrequency catheter ablation for atrial fibrillation: 1-year follow-up study with irrigation tip catheter. J Cardiovasc Electrophysiol. 2014; 25: 693-700.

7 Yamada T, Yoshida N, Murakami Y, et al. Vagal modification can be a valid predictor of late recurrence of paroxysmal atrial fibrillation independent of the pulmonary vein isolation technique. Circ J. 2009; 73: 1606-1611.

8 Wang K, Chang D, Chu Z, et al. Denervation as a common mechanism underlying different pulmonary vein isolation strategies for paroxysmal atrial fibrillation: evidenced by heart rate variability after ablation. ScientificWorldjournal. 2013; 2013: 569564 .

9 Mujović N, Marinković M, Marković N, et al. Persistency of left atrial linear lesions after radiofrequency catheter ablation for atrial fibrillation: data from an invasive follow-up electrophysiology study. J Cardiovasc Electrophysiol. 2017; 28: 1403-1414.

10 Shaffer F, Ginsberg JP. An overview of heart rate variability metrics and norms. Front Public Health. 2017; 5: 258.

11 Liu P, Guo JH, Zhang HC, et al. Vagal effects on the occurrence of focal atrial fibrillation originating from the pulmonary veins. Circ J. 2009; 73: 48-54.

12 Seaborn GE, Todd K, Michael KA, et al. Heart rate variability and procedural outcome in catheter ablation for atrial fibrillation. Ann Noninvasive Electrocardiol. 2014; 19: 23-33.

13 Yu HT, Kim TH, Uhm JS, et al. Prognosis of high sinus heart rate after catheter ablation for atrial fibrillation. Europace. 2017; 19: 1132-1139.

14 Hillebrand S, Gast KB, de Mutsert R, et al. Heart rate variability and first cardiovascular event in populations without known cardiovascular disease: meta-analysis and dose-response meta-regression. Europace. 2013; 15: 742-749.

15 Sikorska A, Pilichowska-Paszkiet E, Zuk A, et al. Acceleration of sinus rhythm following ablation for atrial fibrillation: a simple parameter predicting ablation efficacy. Kardiol Pol. 2019; 77: 960-965.

16 Hindricks G, Sepehri Shamloo A, Lenarczyk R, et al. Catheter ablation of atrial fibrillation: current status, techniques, outcomes and challenges. Kardiol Pol. 2018; 76: 1680-1686. 